\title{
Continuous-Wave Band Translation Between the Near-Infrared and Visible Spectral Ranges
}

\author{
Rui Jiang, Robert E. Saperstein, Nikola Alic, Member, IEEE, Maziar Nezhad, Member, IEEE, Colin J. McKinstrie, \\ Joseph E. Ford, Yeshaiahu Fainman, Fellow, IEEE, and Stojan Radic, Member, IEEE
}

\begin{abstract}
Wavelength conversion based on degenerate fourwave mixing in a photonic crystal fiber with two zero-dispersion wavelengths is investigated both theoretically and experimentally. The proposed concept of universal band translation in a single-pass traveling-wave structure offers a wavelength band rather than a single-wavelength mapping between distant spectral ranges. Near-infrared signals are modulated using both harmonic and pseudorandom bit sequences and translated to the visible optical band. Multiple-channel translation, which produces wavelength-division-multiplexed idlers in the visible band, is demonstrated for the first time. The performance of the translation process is measured both spectrally and temporally for both single- and multiple-channel signals.
\end{abstract}

Index Terms-Band translation, parametric process, photonic crystal fiber.

\section{INTRODUCTION}

O PTICAL transmission in distant spectral windows is characterized by unique features that are generally not found in the standard telecommunication band at $1550 \mathrm{~nm}$. As an example, a mid-infrared (IR) light (3000-6000 nm) can be efficiently transmitted through a set of low-absorption atmospheric windows [1]. Remote sensing or general spectroscopy in the mid-IR window is particularly important because the vibrational transitions of most molecules of interest occupy this band [2]. On the other end of the optical range, the blue-green visible band $(480-530 \mathrm{~nm})$ is the recognized lowloss transport window in ocean water [3]. Visible light generally has sufficient energy to excite electronic states, which for molecules larger than triatoms are typically weakly fluorescent [1]. Positioned between the visible and mid-IR, a near-IR light $(1550 \mathrm{~nm})$ is capable of nearly lossless propagation in silica fibers, which form the backbone of the modern telecommunication infrastructure [4]. Unfortunately, methods for light generation, manipulation, and reception vary widely across

Manuscript received June 29, 2006; revised November 20, 2006.

R. Jiang, R. E. Saperstein, M. Nezhad, and Y. Fainman are with the Department of Electrical Engineering, University of California, San Diego, La Jolla, CA 92093 USA (e-mail: rjiang@ucsd.edu).

N. Alic and S. Radic are with the Department of Electrical Engineering and the California Institute for Telecommunications and Information Technologies, University of California, San Diego, La Jolla, CA 92093 USA.

C. J. McKinstrie is with Bell Laboratories, Alcatel-Lucent, Holmdel, NJ 07733 USA.

J. E. Ford is with the Photonic Systems Integration Laboratory, Department of Electrical and Computer Engineering, University of California, San Diego, La Jolla, CA 92093 USA.

Color versions of one or more of the figures in this paper are available online at http://ieeexplore.iee.org.

Digital Object Identifier 10.1109/JLT.2006.888935 these distant bands. Although we can amplify, modulate, and receive high-speed 1550-nm signals, these operations are either difficult or impractical in the visible and mid-IR bands. Indeed, no equivalents of erbium-doped fiber amplifiers (EDFAs), fast modulators, or receivers exist outside the near-IR window. The overwhelming superiority of near-IR technology is the result of three decades of communications research, which is unlikely to be replicated in any other spectral range in the near future.

Instead of developing new band-specific technologies, a universal band translator (UBT) would naturally leverage 1550-nm technology across the entire optical spectrum. A general spectrally invariant communication link could be constructed by using 1550-nm sources and modulators, followed by a band translation to the target (transport) window and reverse translation back to the $1550-\mathrm{nm}$ band for reception and decoding. Similarly, a general sensing architecture would translate light from arbitrary optical windows to take advantage of low-noise 1550-nm amplifiers and detectors.

Parametric processes are the bases for spectrally invariant UBT technology extending from the visible to the IR. One such process, $\chi^{(3)}$ photon exchange in silica, offers subpicosecond response times, and strict phase and quantum-state preservation over a wide spectral range [5], [6]. The weak Kerr response of silica can be compensated by long phase-matched interaction regions and high waveguide-confinement factors [7]. Parametric signal processing in fibers has been revitalized by recent advances in the manufacturing of highly nonlinear fiber (HNLF). HNLF offers parametric amplification and conversion efficiencies [7], [8] in excess of $50 \mathrm{~dB}$ in the immediate vicinity of the 1550-nm band. Unfortunately, the classical step-index design of HNLF does not support single-mode guidance far from $1550 \mathrm{~nm}$. This deficiency can, in principle, be corrected by silica photonic-crystal-fiber (PCF) structures, which simultaneously guide spectrally distant signal, pump, and idler bands from 400 to $2000 \mathrm{~nm}$. Phase-matched photon exchange in the PCF offers high power efficiency and strict phase fidelity over a wide spectral range [9], [10]. The wavelength-conversion (WC) efficiency generally depends on peak pump power, pumpsignal interaction length, and optical nonlinearity [6], [8]. In contrast to conventional rules which apply to the pulsed regime [13], a UBT peak pump power cannot be arbitrarily scaled: Format transparency, which is required for genuine band translation, dictates the operation in the continuouswave $(\mathrm{CW})$ regime. Just as important, the pump-signal interaction length is dictated by the choice of medium: Crystalline 
materials, while highly nonlinear, can be engineered only to the centimeter scale [16], [17]. An attempt to increase the effective interaction length by enclosing the nonlinear crystal within a resonant cavity effectively limits the translated bandwidth [18].

Transverse PCF engineering makes it possible to synthesize nearly arbitrary dispersion profiles, which are required for phase matching between distant signal and pump waves [9]-[11]. More importantly, a rigorous dispersion control is combined with high confinement, providing an elevated nonlinear response $(\gamma)$. Sharping et al. [13] used these unique characteristics in the construction of an optical parametric oscillator operating in the pulsed regime. Andersen et al. [14] recently demonstrated a $\mathrm{CW}$ conversion of high-power multiple-mode 1493-nm and single-mode 1549-nm signals in the PCF using a tunable Ti:Sapphire pump. The silica PCF platform has reached maturity, with a significant progress made in understanding its guiding properties [12], [15], nonlinear enhancement supporting four-photon mixing (FPM) [14], [20], self- and cross-phase modulations [19] and supercontinuum generation [20], [21]. However, little or no effort has been invested to date [22] in distant modulated signal translation using either silica or nonsilica PCFs.

This paper reports a band-translation study based on the FPM driven by one pump in a PCF with two zero-dispersion wavelengths (ZDWs). A theoretical study of practical PCF parameters was performed in complement to the experimental demonstration of a 1550-nm modulated signal to the visible (500-nm) band. The experiment was performed by the phase matching commercially available 800 -nm pump, multiple telecom band (1550-nm) channels, and submarine communication band $(500 \mathrm{~nm})$. The performance of single- and multiplechannel [wavelength division multiplexing (WDM)] translation to the visible band is measured and quantified spectrally and temporally. Continuous tuning of the modulated visible idler was achieved by a combined control of $800-\mathrm{nm}$ pump and 1550-nm signal.

This paper is organized in four sections: Section II contains the coupled-mode description of the FPM process in PCF, introduces the phase-matching conditions for high-confinement fiber, and compares the conventional HNLF and PCF platforms. Section III describes a set of experiments, which were performed to demonstrate the translation of single- and multiplechannel modulated signals from the $1550-\mathrm{nm}$ band to the 500-nm band. Finally, in Section IV, the main results of this paper are summarized.

\section{One-Pump Parametric Process In PCF}

FPM processes in nonlinear fibers with variety of designs have been investigated in detail during the past 20 years [5], [7]. Degenerate FPM, which is driven by one pump [8], represents the simplest conversion configuration: Two photons from the same pump are annihilated, and one signal photon (parametric amplification) and one idler photon (frequency conversion) are created, provided that both the energy and momentum of the four-photon system are conserved. The quantum effect ensures high-energy efficiency in near-IR to visible translation: Approximately two thirds of the pump power is used to generate the short-wavelength (idler) photon, whereas only one third of the power is used to amplify the long-wavelength (signal) photon. One-pump parametric interaction in nonlinear fiber is described by the coupled-mode equations [14]

$$
\begin{array}{r}
\frac{\partial A_{\mathrm{p}}(z)}{\partial z}=i \gamma\left[\left|A_{\mathrm{p}}\right|^{2} A_{\mathrm{p}}+2\left(\left|A_{\mathrm{s}}\right|^{2}+\left|A_{\mathrm{i}}\right|^{2}\right) A_{\mathrm{p}}\right. \\
\left.+2 A_{\mathrm{i}} A_{\mathrm{s}} A_{\mathrm{p}}^{*} \exp (i \Delta \beta z)\right]-\frac{\alpha_{\mathrm{p}}}{2} A_{\mathrm{p}} \\
\frac{\partial A_{\mathrm{s}}(z)}{\partial z}=i \gamma\left[\left|A_{\mathrm{s}}\right|^{2} A_{\mathrm{s}}+2\left(\left|A_{\mathrm{p}}\right|^{2}+\left|A_{\mathrm{i}}\right|^{2}\right) A_{\mathrm{s}}\right. \\
\left.+A_{\mathrm{p}}^{2} A_{\mathrm{i}}^{*} \exp (-i \Delta \beta z)\right]-\frac{\alpha_{\mathrm{s}}}{2} A_{\mathrm{s}} \\
\frac{\partial A_{\mathrm{i}}(z)}{\partial z}=i \gamma\left[\left|A_{\mathrm{i}}\right|^{2} A_{\mathrm{i}}+2\left(\left|A_{\mathrm{p}}\right|^{2}+\left|A_{\mathrm{s}}\right|^{2}\right) A_{\mathrm{i}}\right. \\
\left.+A_{\mathrm{p}}^{2} A_{\mathrm{s}}^{*} \exp (-i \Delta \beta z)\right]-\frac{\alpha_{\mathrm{i}}}{2} A_{\mathrm{i}}
\end{array}
$$

where $\gamma$ is the nonlinear coefficient, $\alpha$ is the loss coefficient, and $A_{\mathrm{p}, \mathrm{s}, \mathrm{i}}$ are the complex field amplitudes of the pump, signal, and idler, respectively. The linear wavenumber mismatch parameter is defined as

$$
\Delta \beta=\beta_{\mathrm{s}}+\beta_{\mathrm{i}}-2 \beta_{\mathrm{p}}
$$

The wavenumber mismatch parameter is defined by the dispersion property of the fiber at spectrally distant pump, signal, and idler wavelengths. The total wavenumber mismatch $\kappa$ includes both the linear and nonlinear contributions:

$$
\kappa=\Delta \beta+2 \gamma P_{0}
$$

where $P_{0}$ is the pump power. In the nondepleted pump interaction, the maximal gain $g=\gamma P_{0}$ is attained in case of vanishing mismatch:

$$
\Delta \beta(\omega)+2 \gamma P_{0}=0 .
$$

Equation (6) defines the wavenumber matching condition of the parametric process. In physical terms, the phase-matching condition describes a momentum conservation during the fourphoton pump-signal-idler exchange. In classical terms, it simply means that the wavevector of the idler has to be matched to the vector of the grating formed by the beating of the pump and the signal. In practical terms, the phase matching (6) can be solved either by treating the linear mismatch contribution $\Delta \beta(\omega)$ as a continuous function of $\omega$ or by using its Taylor expansion and finding the roots of the corresponding polynomial relation [14]. While the latter method is not exact, by incorporating a sufficient number of higher order terms, the arbitrary accuracy solution can be found. This approach is particularly applicable to the PCF with complex dispersion and loss characteristics that are not easily described in analytic terms.

The coupled-mode (1)-(6) were successfully used to describe one-pump parametric interactions in both HNLFs [6]-[8] and PCFs [14]. HNLF, having a conventional step-index structure, usually features a single ZDW and a nearly flat dispersion 


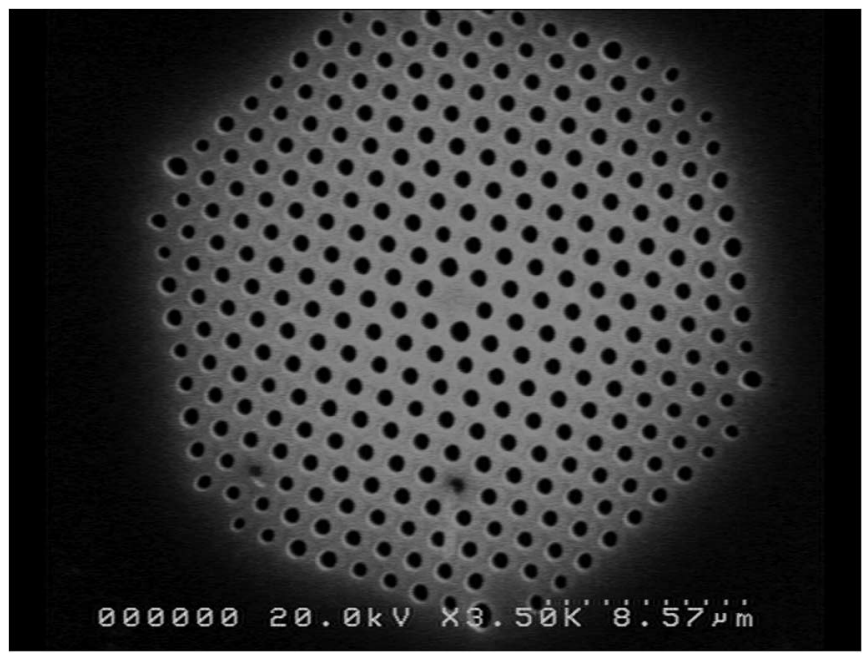

Fig. 1. Scanning electron microscope image of the commercial photonic crystal fiber used in this paper.

curve near the ZDW. The FPM in this region can be phase matched by balancing the linear wavenumber shift $(\Delta \beta)$ and the nonlinear wavenumber shift $(2 \gamma P)$. Since the dispersion slope is small $\left(S \sim 0.03 \mathrm{ps} / \mathrm{km} / \mathrm{nm}^{2}\right)$, only the second-order dispersion term is significant, and the effects of high-order dispersion terms are negligible within a spectral range that encompasses the conventional C- and L-bands. Consequently, the solution of the coupled-mode equations is relatively simple and even in the case of heavily depleted pumps, can be found in a straightforward manner [6]. However, the 1000-nm translation range considered in this paper eliminates the use of HNLF since its step-index design cannot support the dispersion engineering over this wide range.

In contrast, the PCF offers qualitatively different capabilities in precise dispersion tailoring across a near-arbitrary spectral range. The PCF structure used in this paper is a standard commercial product designed for supercontinuum generation at $800 \mathrm{~nm}$ and consists of a hexagonal silica core with a 1.6- $\mu \mathrm{m}$ diameter, surrounded by a cladding with a honeycomb arrangement of circular air holes, as illustrated by the micrograph shown in Fig. 1. By controlling the ratio of the airhole size and adjacent cell distance, it is possible to support a single-mode propagation in bands separated by hundreds of nanometers [15]. More importantly, the variation of the same parameters can be used to synthesize the balanced higher order dispersion terms and achieve a phase matching for spectrally distant signal-pump-idler waves [14], [23].

Fig. 2(a) shows the dispersion curve of the PCF used in the experiment. The PCF is characterized by two ZDWs, at 750 and $1260 \mathrm{~nm}$, and a maximum dispersion of $69.5 \mathrm{ps} / \mathrm{km} \cdot \mathrm{nm}$ at $1001 \mathrm{~nm}$. The nonlinear parameter was estimated to be $70 \mathrm{~W}^{-1} \cdot \mathrm{km}^{-1}$ near $800 \mathrm{~nm}$. The large curvature of the dispersion curve requires the inclusion of terms up to the eighth order in the corresponding Taylor expansion, in order to provide an accurate description of the phase matching and parametric interaction.

Fig. 2(b) plots the phase-matching contour corresponding to the dispersion curve in the case of negligible nonlinear phase mismatch, in agreement with the experimental condition of this paper. By tuning the pump, which is indicated by the vertical dashed line, two intersections with phase-matching loci define paired signal and idler wavelengths. A commercial 780-nm diode pump allows the standard telecommunication band at $1550 \mathrm{~nm}$ to be matched with the submarine communication window at $500 \mathrm{~nm}$. We note that the ideal pairing of signal and idler waves on the phase-matching contour guarantees the maximal conversion efficiency, even in the case of low pump powers. As the pump power increases, the phase-matching condition can also be altered by the interplay of linear and nonlinear phase shifts in the region near the pump wavelength, as the regular modulation-instability (MI) contribution, positioned close to the pump wavelength, becomes important. Since the translation-paired sidebands, defined by 1550 - and $500-\mathrm{nm}$ waves in this case, are much farther from the pump wavelength than those affected by the MI, their center wavelength as well as their spectral shape is less sensitive to the pump-power fluctuations [14]. On the other hand, for the same reason, the outer sidebands are very sensitive to the dispersion fluctuations, which tend to be significant for fibers with such small cores.

The performance of the translator is defined in a significant way by the effective bandwidth [10], [11]. While the translation of low-rate single-channel communication can, in principle, be accomplished by cavity-aided crystalline devices [16], [17], a general UBT should be capable of mapping the entire WDM band. The effective bandwidth of the PCF translator can be controlled by a precise tailoring of local PCF dispersion curvatures at signal, pump, and idler wavelengths. An important design consideration is related to the high PCF dispersion experienced by the signal modulated at a high rate. Indeed, a signal modulated at gigabits per second or faster is expected to sustain a high intersymbol interference (ISI) in a highdispersion waveguide. While it is possible, at least in principle, to synthesize the dual-ZDW PCF to simultaneously provide spectrally distant phase matching and limited local dispersion at the signal (idler) bands, it is unlikely that this approach would be required for any practical data rates. The high PCF nonlinear parameter $\left(\gamma \sim 100 \mathrm{~W}^{-1} \cdot \mathrm{km}^{-1}\right)$ dictates the use of relatively short fiber segment $(<20 \mathrm{~m})$, which limits the total dispersion within the signal band to approximately $3 \mathrm{ps} / \mathrm{nm}$. This small but finite dispersion is beneficial at practical channel rates $(<40 \mathrm{~Gb} / \mathrm{s}$ ) since it alleviates constraints on the WDM signal grid: The channel spacing can be made considerably smaller than that in the HNLF-based system while maintaining a low interchannel FPM crosstalk.

\section{EXPERIMENTS AND DISCUSSIONS}

A one-pump parametric device was constructed as shown in Fig. 3. A single-frequency CW Ti:Sapphire laser, which is tunable between 780 and $840 \mathrm{~nm}$, served as a parametric pump and operated in the anomalous dispersion region of the PCF coil. The polarization-dependent nature of the PCF required the use of a half-waveplate (W1) to align the pump polarization to a principal axis of the PCF. A dichroic beam splitter (M2) was used to combine the pump and signal $(1550 \mathrm{~nm})$ bands into a high-numerical-aperture (NA) coupling objective (MO1). A 
(a)

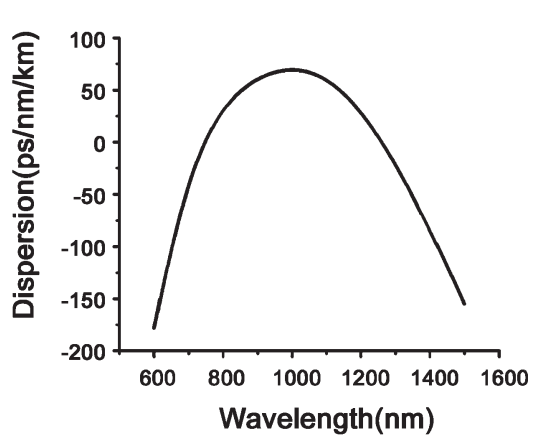

(b)

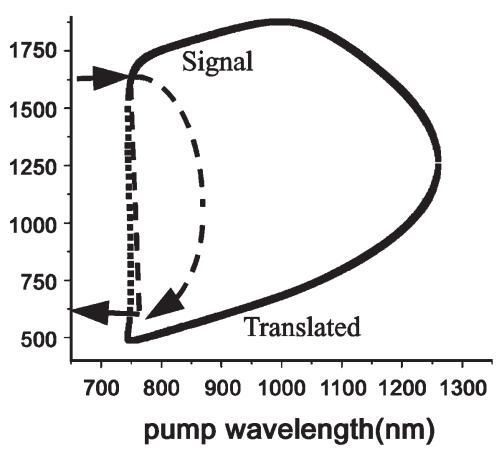

Fig. 2. (a) Dispersion curve of the PCF with two ZDWs at 750 and $1260 \mathrm{~nm}$, respectively. (b) Phase-matching contour of the PCF shown on the right.

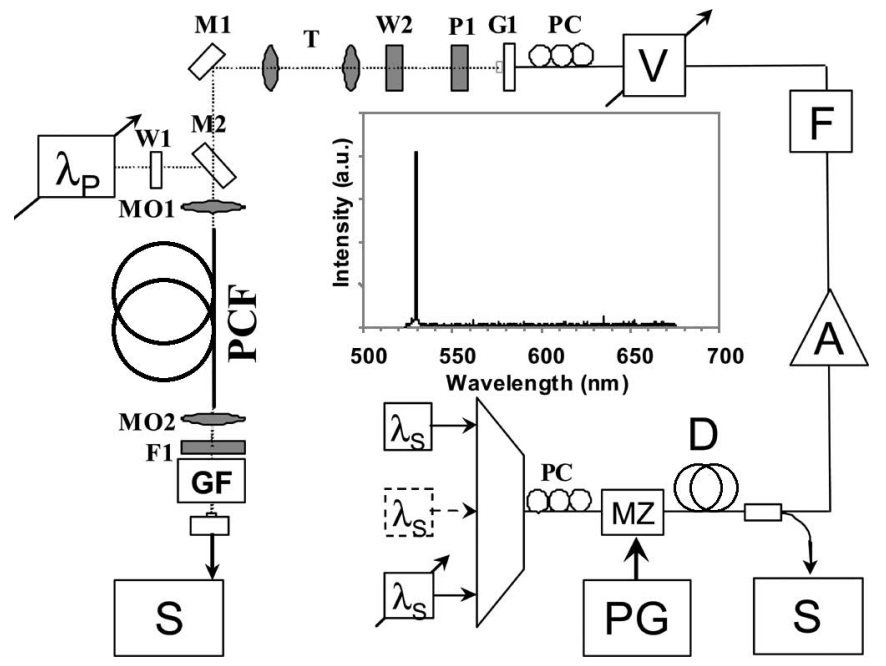

Fig. 3. Experimental setup; symbols as defined in text. Inset indicates typical visible (converted) single-channel spectrum. PC-polarization controller; MZ-Mach-Zehnder amplitude modulator; PG-pattern generator; D-fiber decorrelator; S-scope; A-amplifier; F-filter; V-variable optical attenuator; P1-polarizer; W1-W2- $\lambda / 2$ waveplate; T-telescope; M1—metallic mirror; M2-Dichroic mirror; MO1-MO2-microscopic objective; GF-grating filter.

standard microscopic objective optimized for visible transmission was used here, and the transmission for both the pump and signal was only $75 \%$. Ideally, an IR objective should be used.

The first set of experiments was performed using a single external cavity laser signal source tuned from 1530 to $1600 \mathrm{~nm}$. The source was modulated using a Mach-Zehnder (MZ) modulator driven by either a harmonic or a pseudorandom pattern generator, with varied pattern length (from $2^{7}-1$ to $2^{31}-1$ bits) and modulation speeds of 50, 155.52, 200, and $400 \mathrm{MHz}$. The initial modulation speed was limited by the slow response (100-MHz bandwidth) of the visible detector. The modulated signal was boosted $(\mathrm{A})$ and filtered $(\mathrm{F})$ in order to reject excess amplified spontaneous emission from the EDFA. A fiber polarization controller (PC) was used to maximize the gradient-index lens (G1) coupling to the free-space signal arm (G1-M1). The combination of a linear polarizer (P1) and a half-wave plate (W2) was used to align the pump and signal polarizations prior to the insertion in the $\mathrm{PCF}$, with minimal coupled powers of 20.5 and $10 \mathrm{dBm}$, respectively, throughout the experiment. A dedicated telescope (T) segment collimated the signal beam before it was coupled into the PCF by the objective (MO1). A metallic mirror (M1) was used to steer the signal arm to overlap with the pump. Two 20-m coils of PCF that have the same claimed dispersion curve were used to compare the performance. The PCF output was collimated using a second objective (MO2 40×) and filtered by a 600-nm low-pass dichroic element (F1), which separated the idler band from the pump and the signal bands. All the waves were observed in fundamental mode at the PCF output. The final (visible) idler had a sufficient power in all the experimental configurations (single channel and WDM) to overcome the output coupling losses (MO2, F1) and was well above the sensitivity threshold of the receiver. In the second set of experiments, the single tunable source was replaced by four WDM channels, which were modulated and decorrelated prior to the booster (A). The visible channels were then dispersed by a grating filter (GF), selected by a spatial filter, and detected by either a fast oscilloscope or the visible detector. In both sets of experiments, the input signal channels were monitored (C) prior to the booster stage using a fast oscilloscope $(\mathrm{S})$. In the last set of experiments, the $\mathrm{Ti}$ : Sapphire pump was replaced by a CW diode laser with a high output power $(\sim 1 \mathrm{~W})$ tunable over 768-786 nm. A faster (1-GHz bandwidth) but less sensitive receiver was used in this set of experiments. The translation of a $2^{31}-1$ pseudorandom bit sequence (PRBS) pattern modulated at $1-\mathrm{Gb} / \mathrm{s}$ rate, to a $500-\mathrm{nm}$ band idler, was observed in an errorfree manner.

The experimental performance of the translator, particularly in terms of conversion efficiency, was limited by the fact that pump, signal, and idler waves were not exactly phase matched. The phase-matching contour, which was obtained from approximate manufacturer's dispersion data, shown in Fig. 2(b), indicates that a 780-nm pump needs to be paired with a $1550-\mathrm{nm}$ signal. The Ti:Sapphire pump-power peak was offset from this wavelength, dictating an operation in $800-\mathrm{nm}$ vicinity. This departure from the ideal phase matching is further compounded by the fact that the commercial PCF sample was not designed to guide the standard 1550-nm telecom band. These experimental constraints were investigated using the coupledmode (1)-(3) with an excessive 1550-nm band loss. Fig. 4 compares the typical power evolution estimates along the fiber for ideal (lossless) and lossy PCF samples. Relatively low pump power $(20 \mathrm{dBm})$ and a weak input signal $(10 \mathrm{dBm})$ lead to a 


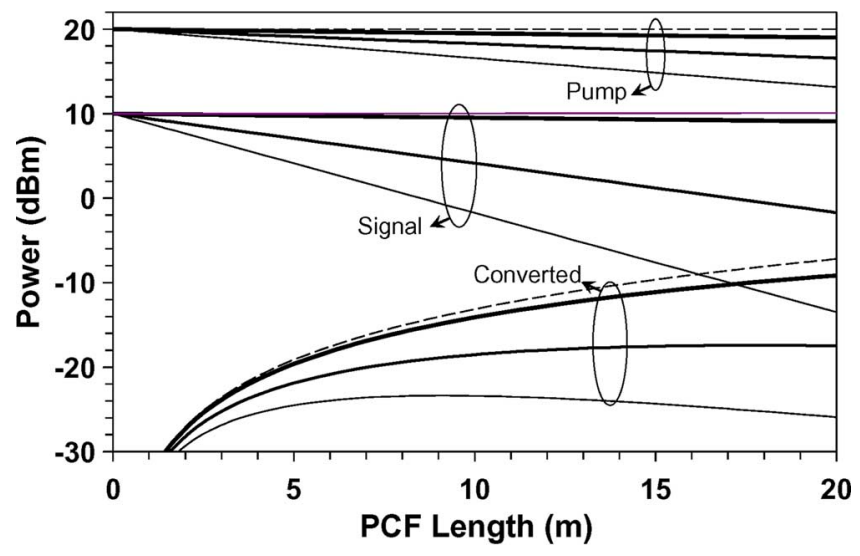

Fig. 4. Pump (800 nm), signal $(1550 \mathrm{~nm})$, and idler $(539 \mathrm{~nm})$ evolution in case of lossless (dashed curve) and lossy PCF. Corresponding losses for signal/pump/idler bands in decibel per kilometer: 50/50/50 (heavy curve), 600/175/200 (medium curve), and 1200/350/400 (thin curve).

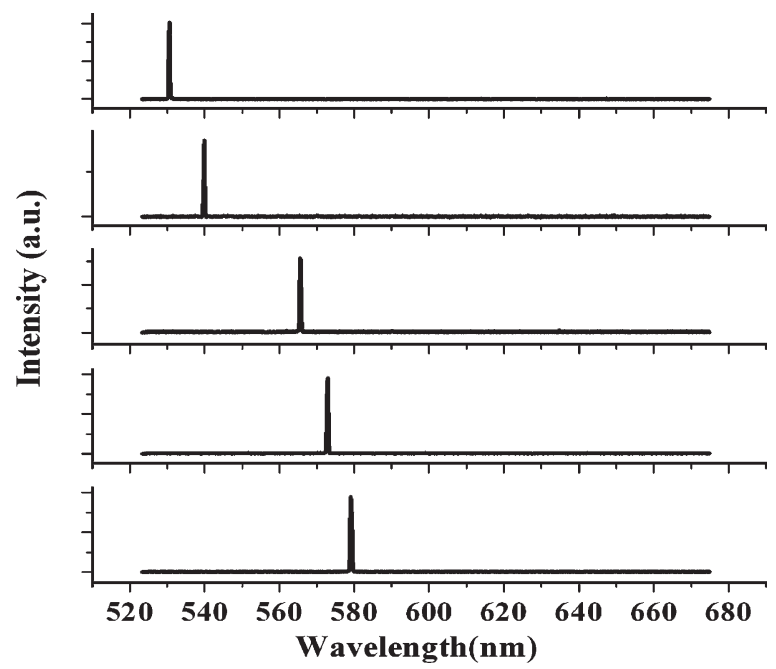

Fig. 5. Typical idler spectrum tuned across the visible band and measured by the visible spectrum analyzer.

$-7-\mathrm{dBm}$ translated wave at $500 \mathrm{~nm}$. A moderate loss of $50 \mathrm{~dB} / \mathrm{km}$ across all three bands decreases the conversion efficiency by $2 \mathrm{~dB}$, as indicated by the thick curve. This case is of particular practical interest since it points to the operational efficiency that could be attained by a current PCF manufacturing process. Any loss in excess of $50 \mathrm{~dB} / \mathrm{km}$ has a significant impact on the translation efficiency, even for the short sample lengths used in this experiment: A total signal loss of $10 \mathrm{~dB}$ or more reduces the converted power to below $-20 \mathrm{dBm}$. In the experiment, a $-25-\mathrm{dBm} 500-\mathrm{nm}$ idler was converted from a $10-\mathrm{dBm} 1550$ signal, resulting in a conversion efficiency similar to that reported in [14]. Fig. 5 illustrates a typical spectrum obtained by a visible-range spectrometer recorded with a $0.5-\mathrm{nm}$ resolution. The idler was tuned across the visible range to demonstrate the available translator bandwidth. The first coil under investigation allowed a continuous tuning in the ranges of $515-525 \mathrm{~nm}$ and 530-542 nm, whereas the second coil covered the ranges of $515-525 \mathrm{~nm}$ and $565-580 \mathrm{~nm}$. The observed gaps in the continuous tuning range are attributed to the fact that the Ti:Sappire laser exhibited large power variations during the tuning process. We observed Ti:Sapphire pump-power fluctuations (a)

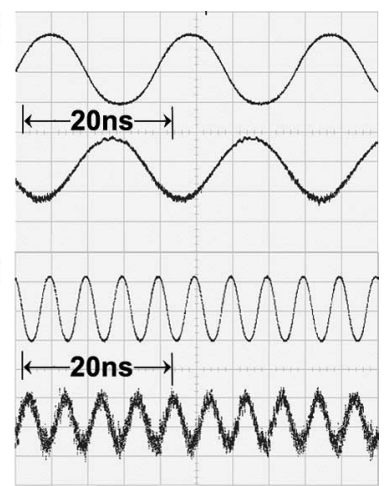

Fig. 6. (a) Fifty-megahertz harmonically modulated 1541-nm signal (upper trace, 1550-nm PIN receiver) and its translated $(531 \mathrm{~nm}$ ) waveform (lower trace) and (b) 200-MHz harmonically modulated 1541-nm signal (upper trace) and its translated waveform, corresponding to $3-\mathrm{dB}$ frequency rollover point of the visible receiver.

of nearly $30 \%$ occurring across the nanometer-scale tuning of the pump wavelength, thus preventing a rigorous characterization of the equalized translator bandwidth. The idler power exhibited a high sensitivity to the input pump polarization, which is in agreement with the previous observation [14]. The rotation of the half-waveplate (W1) within the pump arm was used to demonstrate nearly complete idler extinction.

The first modulation experiment was performed with the pump and signal set at 790 and $1541 \mathrm{~nm}$, respectively; the signal was modulated harmonically. The generated visible idler at $531 \mathrm{~nm}$ was received by a visible detector, with a cutoff frequency of $100 \mathrm{MHz}$, and was analyzed on the oscilloscope, as illustrated in Fig. 6. Although the signal was modulated at rates up to $10 \mathrm{~Gb} / \mathrm{s}$, the absence of a fast visible detector rendered any faster measurements $(>200 \mathrm{Mb} / \mathrm{s}$ or $1.5 \mathrm{~Gb} / \mathrm{s}$ in the later experimental stage) impossible. Modulated and translated waveforms at 50 and $200 \mathrm{MHz}$ are shown in Fig. 6(a) and (b), illustrating the fundamental limitation posed by the slow receiver. The measured idler power scaled linearly with the signal power, as expected for a nondepleted one-pump parametric interaction [14]. The translated modulation measurement was dominated by the frequency response of the receiver, as illustrated in Fig. 6. In the case of 50-MHz harmonic modulation, the recovered idler exhibited a high extinction ratio, with average low and high logic levels of 1 and $500 \mathrm{mV}$, respectively. Fast harmonic modulation at $200 \mathrm{MHz}$ exceeded the 3-dB receiver rollover by many tens of megahertz, resulting in the distortion illustrated in Fig. 6(b).

A second set of modulation experiments was performed using a nonreturn-to-zero (NRZ) PRBS in order to estimate the penalty induced by the translation process. The modulation rate was varied from 50, 155.52 (OC-3), and $400 \mathrm{Mb} / \mathrm{s}$, with short $\left(2^{7}-1\right)$ and long $\left(2^{31}-1\right)$ bit sequences. The idler waveforms were analyzed using both the sampling oscilloscope and the bit-error-rate (BER) analyzer. Fig. 7 illustrates typical received idler patterns at $2^{7}-1$ pattern length. Both 50and $155.52-\mathrm{Mb} / \mathrm{s}$ patterns are nearly unimpaired, whereas the $400-\mathrm{Mb} / \mathrm{s}$ pattern exhibits a heavy ISI imposed by insufficient receiver bandwidth. Our PRBS modulation test was thereafter performed at $155.52 \mathrm{Mb} / \mathrm{s}$. Fig. 8 shows a typical eye diagram with a measured $Q$-factor of $15.23 \mathrm{~dB}$ at $155.52 \mathrm{~Gb} / \mathrm{s}$. This 
(a)

(b)

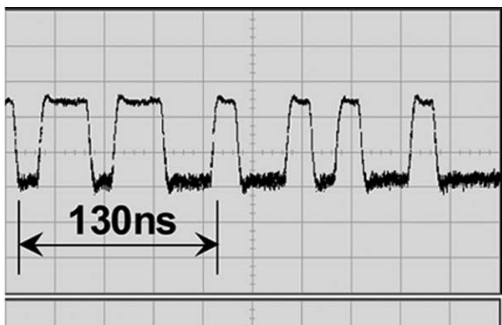

(c)
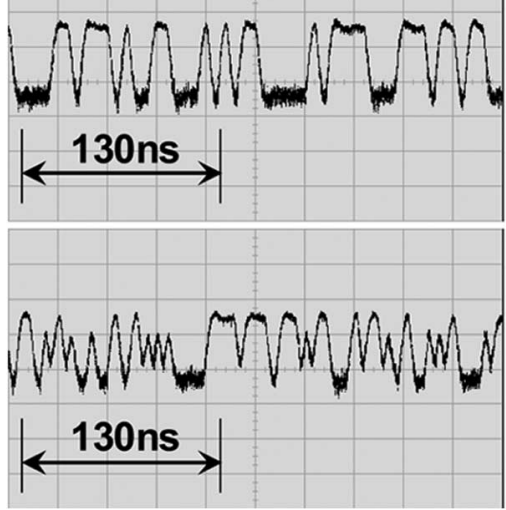

Fig. 7. (a) Fifty-Mb/s, (b) $155.52-\mathrm{Mb} / \mathrm{s}$, and (c) 400-Mb/s modulated PRBS words received at $531 \mathrm{~nm}$.

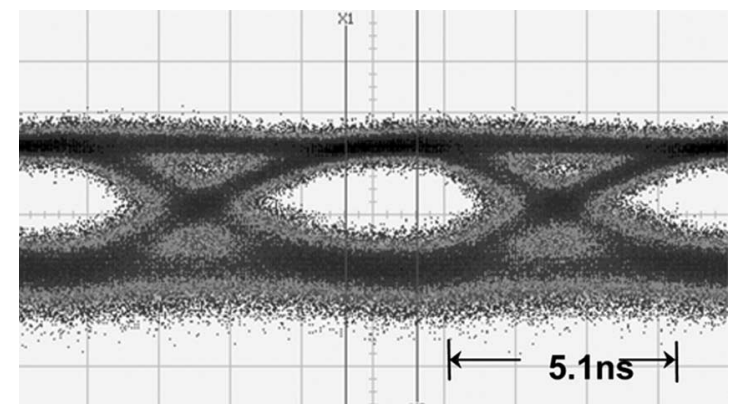

Fig. 8. Eye pattern $(531 \mathrm{~nm})$ with $155.52-\mathrm{Mb} / \mathrm{s}$ NRZ modulation. The eye penalty is dominated by the operation of the visible receiver beyond 3-dB rollover point.

translated performance incorporates the cumulative penalty associated with the operation of the visible receiver beyond its 3-dB rollover point, the noisy Ti:Sapphire pump, and the excessive PCF loss at $1550 \mathrm{~nm}$. The BER measurements of short PRBS words $\left(<2^{10}-1\right)$ indicated an error-free performance; however, the longest PRBS sequence $\left(2^{31}-1\right)$ pattern had a measured BER of $6 \times 10^{-8}$. This degradation increase is attributed to the patterning effect that originates from the unequalized frequency response of the detector, particularly near the dc region.

In addition to the translation of a single modulated channel at $1550 \mathrm{~nm}$, we explored the feasibility of WDM (band) translation. Four channels positioned at 1541, 1549, 1555, and $1560 \mathrm{~nm}$ were translated using a fixed and tunable Ti:Sapphire pump. Fig. 9 illustrates a WDM band casting corresponding to the parametric pumps positioned at 791,800 , and $802 \mathrm{~nm}$. The longest wavelength channel $(1560 \mathrm{~nm})$ is mapped to either $529 \mathrm{~nm}$ (pump at $791 \mathrm{~nm}$ ) or $540 \mathrm{~nm}$ (pump at $802 \mathrm{~nm}$ ). Parametric WDM upconversion results in a considerably denser channel spacing, with the highest frequency channel separation just resolved by the visible spectrometer used in this setup. A 9-nm wavelength separation in the communication band was mapped to a 1-nm separation in the visible band. It is interesting to note that the ultradense WDM channel spacing of $0.2 \mathrm{~nm}$ $(25 \mathrm{GHz})$ would correspond to a separation of only $0.02 \mathrm{~nm}$ in the visible band, which is well beyond the capabilities of the spectrometer used in this paper. Downconversion back to the $1550-\mathrm{nm}$ band at the receiving end would ease the need for a high-resolution visible spectrometer.

The pump was then placed at $840 \mathrm{~nm}$, and two visible channels converted from two WDM channels at 1565.5 and $1551 \mathrm{~nm}$, respectively, were spectrally demultiplexed, as illustrated in Fig. 10, with the measured $Q$-factors of $10.9 \mathrm{~dB}$. The measurements indicate no significant crosstalk, which is in agreement with the modeled prediction.

In the final set of measurements, the Ti:Sapphire pump and $100-\mathrm{MHz}$ visible receiver were replaced by a CW diode pump and a gigahertz visible receiver, respectively. Faster modulation measurements were obtained using 20.7- $\mathrm{dBm}$ pump and 19-dBm signal coupled powers, yielding $-16.7 \mathrm{dBm}$ of visible power from the 5-m long PCF segment. The idler wavelength was tuned from 512 to $520 \mathrm{~nm}$ by sweeping the pump wavelength, similar to the Ti:Sapphire procedure described above. The NRZ modulated signal translation was thereafter demonstrated by setting the pump at 777.6 and $775.5 \mathrm{~nm}$, respectively, and the signal at $1575 \mathrm{~nm}$, resulting in idler wavelengths of 516.2 and $514.4 \mathrm{~nm}$. The idler was received by the visible detector, which has a 3-dB rollover frequency of $1 \mathrm{GHz}$, and was subsequently observed using the fast sampling scope. The received $1-\mathrm{Gb} / \mathrm{s}$ NRZ waveform and eye diagram are shown in Fig. 11(a). The corresponding $Q$-factor is $15.27 \mathrm{~dB}$. The performance of the architecture was quantified by the BER, measured using a $2^{31}-1$ PRBS pattern to achieve an error-free performance. Fig. 12 shows the measured BER curve at the two different wavelengths, indicating that a tuning did not influence the performance of the WC process. By increasing the NRZ rate beyond the receiver $3-\mathrm{dB}$ rollover frequency $(1 \mathrm{GHz})$, the amplitude of the $1.5-\mathrm{GHz}$ idler waveform was reduced and the measured $Q$-factor dropped to $13.26 \mathrm{~dB}$, as shown in Fig. 11(b).

\section{CONCLuSION}

In summary, we have demonstrated the translation of single and multiple modulated channels between the standard nearIR and visible bands. The reported conversion over $375 \mathrm{THz}$ is a record for modulated channel translation in an all-fiber structure. BERs at $155 \mathrm{Mb} / \mathrm{s}$ and $1 \mathrm{~Gb} / \mathrm{s}$ were measured for the intensity modulated signals translated from the near-IR to the visible spectrum. No significant impairment associated with the translation process was observed in either the singleor multiple-channel case. The experimental investigation was performed using commercial PCF coils, which were not designed to support a 1550-nm guiding. Improved translation performance can be achieved by a combination of dispersionoptimized, low-loss PCF, and higher power single-frequency pump, which represents a natural extension of the reported work. While a more sensitive and faster visible detector would certainly facilitate the optimization of the near-IR to visible 

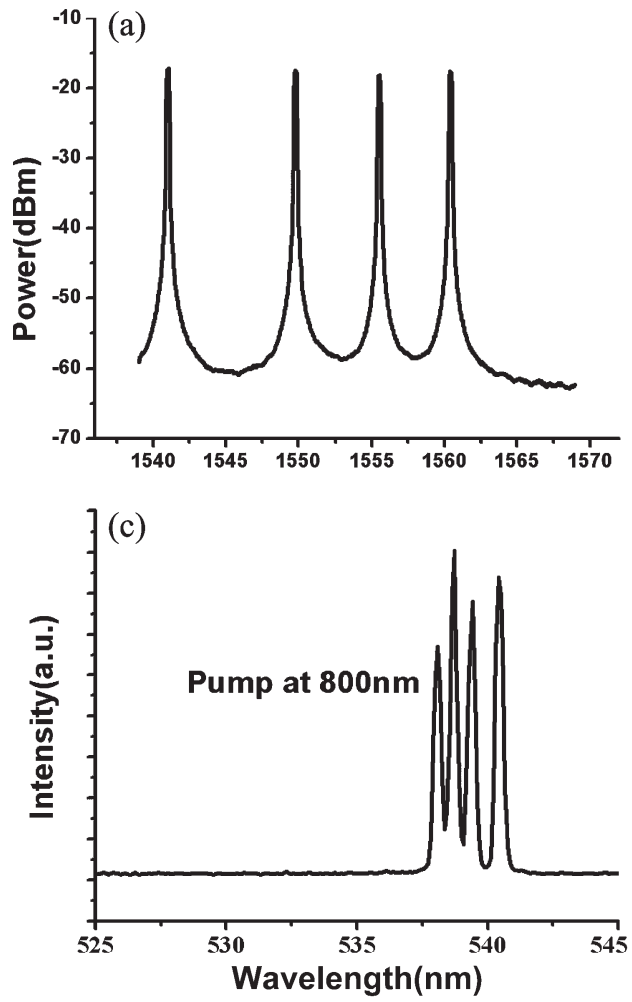

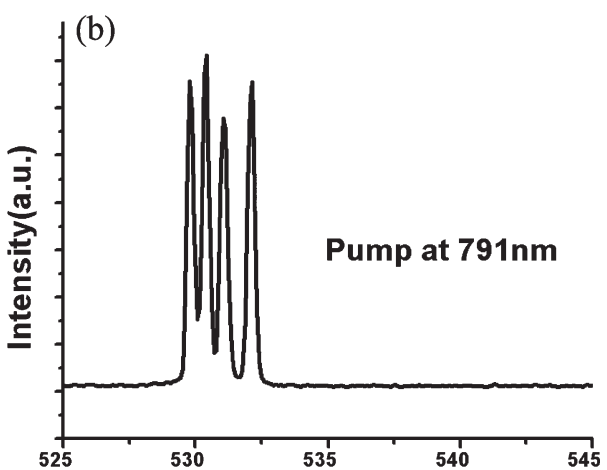

(d)

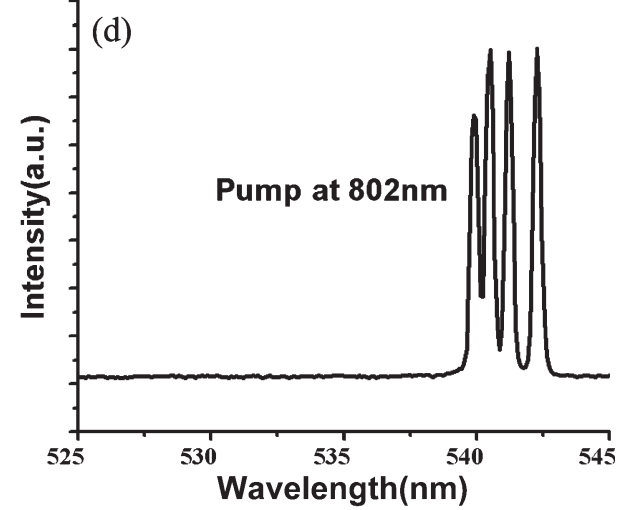

Fig. 9. (a) Four WDM channels at $1541,1549,1555$, and $1560 \mathrm{~nm}$ are translated to visible spectral domain. Parametric pump is tuned to (b) 791 , (c) 800 , and (d) $802 \mathrm{~nm}$.

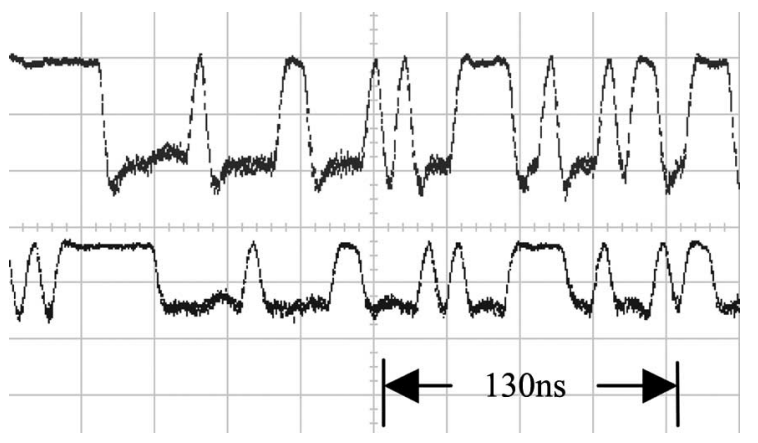

Fig. 10. Channels demultiplexed after a visible translation: $574 \mathrm{~nm}$ (upper trace) and $576 \mathrm{~nm}$ (lower trace). (a)

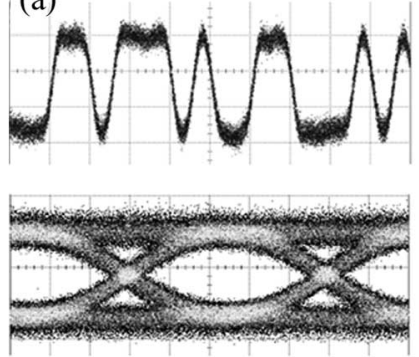

(b)
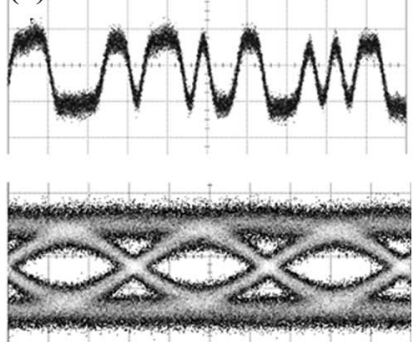

Fig. 11. Measured waveform at (a) 1 and (b) $1.5 \mathrm{~Gb} / \mathrm{s}$ and corresponding eye diagrams. Each division corresponds to $2 \mathrm{~ns}$ for upper figures and $200 \mathrm{ps}$ for lower figures.

translator, its availability (or lack thereof) is not a fundamental obstacle: A two-way time-division architecture can be constructed using the same device, which allows performance

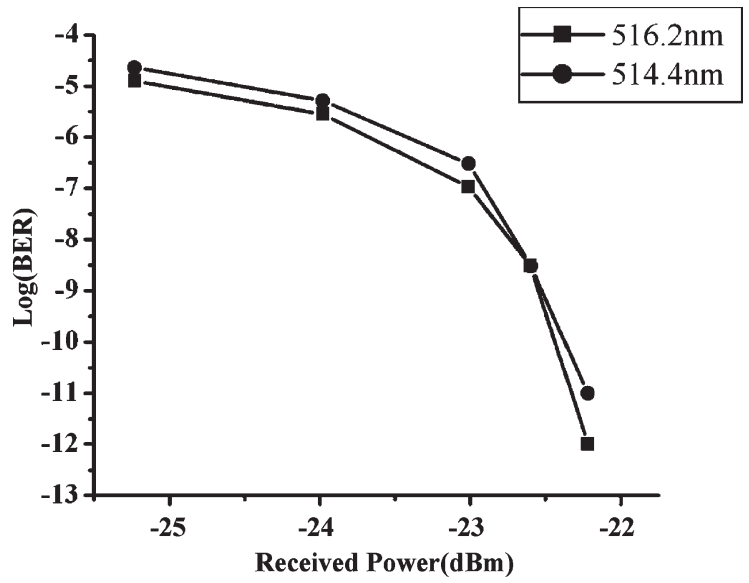

Fig. 12. Measured BER curve for idlers at 516.2 and $514.4 \mathrm{~nm}$, respectively.

characterization within the originating (near-IR) band. We also believe that significant opportunity remains for further investigations that include multiple-pump parametric architectures and dual ZDW-PCFs tailored for nondegenerate FPM coupling. However, we recognize a significant challenge in quantifying the impact of spatial dispersion and polarization fluctuations in the PCF on the performance of the translator system.

\section{ACKNOWLEDGMENT}

The authors would like to thank S. Mookherjea for his help in obtaining the scanning-electron-microscope image of the photonic crystal fiber. 


\section{REFERENCES}

[1] C. N. Banwell and E. M. McCash, Fundamentals of Molecular Spectroscopy, 4th ed. London, U.K.: McGraw-Hill, 1994.

[2] G. M. Gibson, M. H. Dunn, and M. J. Padgett, "Application of a continuously tunable, $\mathrm{cw}$ optical parametric oscillator for high-resolution spectroscopy," Opt. Lett., vol. 23, no. 1, pp. 40-42, Jan. 1998.

[3] J. E. Tyler, Light in the Sea. Strounburg, PA: Dowden (Hithinsons and Ross), 1977

[4] Optical Fiber Telecommunications IV B: Systems and Impairments, I. P. Kaminow and T. Li, Eds. New York: Academic, 2002.

[5] R. H. Stolen and J. E. Bjorkholm, "Parametric amplification and frequency conversion in optical fibers," IEEE J. Quantum Electron., vol. QE-18, no. 7, pp. 1062-1072, Jul. 1982.

[6] C. J. McKinstrie, S. Radic, and A. R. Chraplyvy, "Parametric amplifiers driven by two pump waves," IEEE J. Sel. Topics Quantum Electron., vol. 8, no. 3, pp. 538-547, May/Jun. 2002.

[7] S. Radic and C. J. McKinstrie, "Optical amplification and signal processing in highly nonlinear optical fiber," IEICE Trans. Electron., vol. E88-C, no. 5, pp. 859-869, 2005.

[8] J. Hansryd and P. Andreksen, "Broad-band continuous-wave pumped fiber optical parametric amplifier with 49-dB gain and wavelength conversion efficiency," IEEE Photon. Technol. Lett., vol. 13, no. 3, pp. 194-196, Mar. 2001.

[9] P. Russell, "Photonic crystal fibers," Science, vol. 299, no. 5605, pp. 358362, Jan. 2003.

[10] A. Y. H. Chen, G. K. L. Wong, S. G. Murdoch, R. Leonhardt, J. D. Harvey, J. C. Knight, W. J. Wadsworth, and P. S. J. Russell, "Widely tunable optical parametric generation in a photonic crystal fiber," Opt. Lett., vol. 30, no. 7, pp. 762-764, Apr. 2005.

[11] G. K. L. Wong, A. Y. H. Chen, S. G. Murdoch, R. Leonhardt, J. D. Harvey, N. Y. Joly, J. C. Knight, W. J. Wadsworth, and P. S. J. Russell, "Continuous-wave tunable optical parametric generation in a photonic-crystal fiber," J. Opt. Soc. Amer. B, Opt. Phys., vol. 22, no. 11, pp. 2505-2511, Nov. 2005.

[12] K. Nakajima, J. Zhou, K. Tajima, K. Kurokawa, C. Fukai, and I. Sankawa, "Ultrawide-band single-mode transmission performance in a low-loss photonic crystal fiber," J. Lightw. Technol., vol. 23, no. 1, pp. 7-12, Jan. 2005.

[13] J. Sharping, M. Fiorentino, P. Kumar, and R. Windeler, "Optical parametric oscillator based on four-wave mixing in microstructure fiber," $O p t$. Lett., vol. 27, no. 19, pp. 1675-1677, Oct. 2002.

[14] T. V. Andersen, K. M. Hilligsøe, C. K. Nielsen, J. Thøgersen, K. P. Hansen, S. R. Keiding, and J. J. Larsen, "Continuous-wave wavelength conversion in a photonic crystal fiber with two zero-dispersion wavelengths," Opt. Express, vol. 12, no. 17, pp. 4113-4122, Aug. 2004.

[15] T. A. Birks, J. C. Knight, and P. S. J. Russell, "Endlessly single-mode photonic crystal fiber," Opt. Lett., vol. 22, no. 13, pp. 961-963, Jul. 1997.

[16] L. E. Myers and W. Bosenberg, "Periodically poled lithium niobate and quasi-phase-matched optical parametric oscillators," IEEE J. Quantum Electron., vol. 33, no. 10, pp. 1663-1672, Oct. 1997.

[17] S. E. Bisson, K. M. Armstrong, T. J. Kulp, and M. Hartings, "Broadly tunable, mode-hop-tuned $\mathrm{CW}$ optical parametric oscillator based on periodically poled lithium niobate," Appl. Opt., vol. 40, no. 33, pp. 6049-6055, Nov. 2001.

[18] M. Ebrahimzadeh, G. A. Turnbull, T. J. Edwards, D. J. Stothard, I. D. Lindsay, and M. H. Dunn, "Intracavity CW singly resonant optical parametric oscillators," J. Opt. Soc. Amer. B, Opt. Phys., vol. 16, no. 9, pp. 1499-1511, Sep. 1999.

[19] J. S. Y. Chen, G. K. L. Wong, S. G. Murdoch, R. J. Kruhlak, R. Leonhardt, and J. D. Harvey, "Cross-phase modulation instability in photonic crystal fibers," Opt. Lett., vol. 31, no. 7, pp. 873-875, Apr. 2006.

[20] S. Coen, A. H. L. C. Chau, R. Leonhardt, J. D. Harvey, J. C. Knight, W. J. Wadsworth, and P. S. J. Russell, "Supercontinuum generation by stimulated Raman scattering and parametric four-wave mixing in photonic crystal fibers," J. Opt. Soc. Amer. B, Opt. Phys., vol. 19, no. 4, pp. 753-764, Apr. 2002.

[21] W. J. Wadsworth, A. Ortigosa-Blanch, J. C. Knight, T. A. Birks, T.-P. Martin Man, and P. S. J. Russell, "Supercontinuum generation in photonic crystal fibers and optical fiber tapers: A novel light source," J. Opt. Soc. Amer. B, Opt. Phys., vol. 19, no. 9, pp. 2148-2155, Sep. 2002.

[22] R. Jiang, R. Saperstein, N. Alic, M. Nezhad, C. McKinstrie, J. Ford, Y. Fainman, and S. Radic, "375 THz Parametric translation of modulated signal from $1550 \mathrm{~nm}$ to visible band," presented at the Optical Fiber Commun. Conf., Anaheim, CA, 2006, Paper PD16.

[23] K. P. Hansen, J. R. Folkenberg, C. Peucheret, and A. Bjarklev, "Fully dispersion controlled triangular-core nonlinear photonic crystal fiber," presented at the Optical Fiber Commun. Conf., Atlanta, GA, 2003, Paper PD2.
Rui Jiang received the Bachelor's degree in engineering physics from Tsinghua University, Beijing, China, in 2000 and the Master's degree in laser plasma physics from the University of Rochester, Rochester, NY, in 2004. He is currently working toward the Ph.D. degree at the University of California, San Diego, La Jolla.

He has been with the China Institute of Atomic Energy, dealing with laser plasma physics for two years. His interests include nonlinear fiber optics and optical communications.

Robert E. Saperstein received the B.S.E. degree in electrical engineering from Princeton University, Princeton, NJ, in 2001 and the M.S. degree in photonics from the University of California (UC), San Diego, La Jolla, in 2004. He is currently working toward the Ph.D. degree in photonics at UC San Diego.

He has been a member of Prof. Fainman's ultrafast and nanoscale optics group since 2001. His current research interests include signal processing based on temporal/longitudinal spectral decomposition of ultrafast pulses.

Mr. Saperstein is a Student Member of the Optical Society of America.

Nikola Alic (M'06) was born in Belgrade, Serbia. He received the B.Sc. degree in electrical engineering from the Belgrade School of Electrical Engineering, Belgrade, and the M.S. and Ph.D. degrees in photonics from the University of California San Diego (UCSD), La Jolla, in 2001 and 2006, respectively.

After a short period in the "Vinca" Institute of Nuclear Sciences, Belgrade, he took on his graduate studies at the Department of Electrical and Computer Engineering, UCSD. He currently holds a joint position at the Department of Electrical Engineering, UCSD and the California Institute for Telecommunications and Information Technologies, UCSD. His interests include high-speed optical transmission, signal processing for impairment mitigation in high-speed optical communications, coding theory, and nonlinear fiber optics.

Maziar Nezhad (M'00) received M.Sc. degrees in electrical engineering from the University of Alabama, Huntsville (in optics) and Ferdowsi University, Mashad, Iran (in control systems). He is currently working toward the Ph.D. degree in photonics at the University of California, San Diego, La Jolla.

His current research interests include plasmonics, subwavelength optics, optical sensors, and nonlinear optics.

Mr. Nezhad is a member of the IEEE Lasers and Electro-Optics Society and the Optical Society of America.

Colin J. McKinstrie received the B.Sc. degree from the University of Glasgow, Glasgow, U.K., in 1981 and the Ph.D. degree from the University of Rochester, Rochester, NY, in 1986.

From 1985 to 1988, he was a Postdoctoral Fellow at the Los Alamos National Laboratory, Los Alamos, NM, where he was with the Applied Physics Division and the Center for Nonlinear Studies. In 1988, he returned to the University of Rochester as a Professor of mechanical engineering and a Scientist at the Laboratory for Laser Energetics, where his main research interests were plasma-based particle acceleration, laser-plasma interactions, and nonlinear fiber optics. Since 2001, he has been a member of the Technical Staff at Bell Laboratories, Lucent Technologies (now Alcatel-Lucent), Holmdel, NJ, where his research concerns the amplification and transmission of optical pulses in communication systems.

Joseph E. Ford received the M.S. degree in physics from the University of British Columbia, Vancouver, BC, Canada, the M.S. degree in optical engineering from the University of Rochester, Rochester, NY, and the Ph.D. degree from the University of California (UC), San Diego, La Jolla, in 1992.

From 1994 to 2000, he was a member of Bell Laboratories' Advanced Photonics Research Department, where he developed optoelectronics and microoptomechanics for optical communications. From 2000 to 2002, he was with Optical Micro-Machines. In 2002, he became an Associate Professor at the Department of Electrical and Computer Engineering, UCSD, where he leads the Photonic Systems Integration Laboratory. He is a coauthor of 35 refereed journal articles and 45 U.S. patents on optical components and systems. 
Yeshaiahu Fainman (M'93-SM'01-F'03) received the Ph.D. degree from the Technion-Israel Institute of Technology, Haifa, Israel, in 1983.

$\mathrm{He}$ is a Professor of electrical and computer engineering at the Department of Electrical Engineering, University of California (UC), San Diego, La Jolla. He has contributed more than 150 manuscripts in referred journals and more than 250 conference presentations and conference proceedings. His current research interests include optical network systems and devices, with special emphasis on ultrafast information processing with optical nonlinearities and the use of femtosecond laser pulses, near-field phenomena in optical nanostructures and nanophotonic devices, quantum communication, and multidimensional quantitative imaging.

Dr. Fainman is a Fellow of the Optical Society of America (OSA) and the Society of Photo-Optical Instrumentation Engineers. He was a Chair of the IEEE/IEEE Lasers and Electro-Optics Society Subcommittee on Optical Interconnects and Signal Processing from 2004 to 2006 and a General Chair of the OSA Topical Meeting on Nanophotonics for Information Systems in 2005. He also served on numerous conference program committees, organized symposia and workshops, and, between 1993 and 2001, served as a Topical Editor of the Journal of the Optical Society of America: A on Optical Signal Processing and Imaging Science. He was a recipient of the Miriam and Aharon Gutvirt Prize.
Stojan Radic (M'01) received the degree from The Institute of Optics, University of Rochester, Rochester, NY.

He was subsequently a Senior Scientist with Corning Incorporated, a member of the Technical Staff at Bell Laboratories, and the Nortel Networks Chair at Duke University, Durham, NC. He is currently an Associate Professor at the Department of Electrical Engineering and the California Institute for Telecommunications and Information Technologies, University of California, San Diego, La Jolla, leading the Optical Networking Laboratory. His research is focused on parametric processing and its use in all-optical networks.

Prof. Radic currently serves on the CLEO, OFC, and OAA committees and is an Optical Communications Editor for Optics Express. 